\title{
Safe Implementation of Mechanical Thrombectomy in Acute Stroke Patients with Major Arterial Occlusion and Concomitant Subdural Hematoma
}

\author{
Yong-Won Kim, MD', 3,4, Dong-Hun Kang, MD²,3, 4, Yang-Ha Hwang, MD', 4, , \\ Yong-Sun Kim, MD, PhD ${ }^{2,4,5}$, Sung-Pa Park, MD, PhD',
}

Mechanical thrombectomy (MT) alone in cases of large-vessel acute ischemic stroke (AIS) with a concomitant subdural hematoma (SDH) seems feasible and safe, but there's still a lack of evidence in the clinical literature. We report three cases of AIS with SDH, of which MT was performed with successful recanalization and no major changes in SDH: two elderly men with acute traumatic SDH and one man with incidental chronic SDH. (1) A 67-year-old man with acute traumatic SDH in the right side developed right middle cerebral artery (MCA) stroke, (2) a 72-year-old man with chronic SDH in the left side developed right MCA stroke, and (3) a 76-year-old-man with acute traumatic SDH in the right side developed top-of-basilar artery syndrome. As for AIS patients with a SDH, MT may be the only feasible and safe treatment option.

Key Words : Acute ischemic stroke; Subdural hematoma; Mechanical thrombectomy

The treatment options for acute ischemic stroke due to a large vessel occlusion include systemic thrombolytic infusion, endovascular revascularization therapy or both [1]. However, we sometimes encounter

'Department of Neurology, ${ }^{2}$ Neurosurgery, ${ }^{3}$ Radiology, and ${ }^{4}$ Cerebrovascular Center, Kyungpook National University Hospital, Daegu, Korea; ${ }^{5}$ School of Medicine, Kyungpook National University, Daegu, Korea

Received July 2, 2013; accepted after revision May 16, 2013.

Correspondence to: Yang-Ha Hwang, MD, Department of Neurology and Cerebrovascular Center, Kyungpook National University Hospital, 130, Dongduk-ro, Jung-gu, Daegu 700-721, Korea.

Tel. 82.53.420.5758 Fax. 82.53.422.4265

E-mail: yangha.hwang@gmail.com

This is an Open Access article distributed under the terms of the Creative Commons Attribution Non-Commercial License (http://creativecommons.org/licenses/by-nc/3.0) which permits unrestricted non-commercial use, distribution, and reproduction in any medium, provided the original work is properly cited. cases of acute ischemic stroke where there is concomitant intracranial bleeding, e.g., subdural hematoma (SDH). The use of systemic thrombolysis is contraindicated in patients having intracranial hemorrhage evidenced by clinical history or imaging [1]. With regard to endovascular therapy, the use of a lower dose and local delivery of a thrombolytic agent also has a risk of intracranial bleeding [2]. The adoption of mechanical thrombectomy (MT) devices alone may seem to be feasible and safe in cases with concomitant $\mathrm{SDH}$, but there's still a lack of evidence in the clinical literature. We report three patients with acute ischemic stroke having a concomitant SDH who were treated by MT alone.

\section{CASE REPORTS}

Between November 2009 and March 2011, we 
performed endovascular revascularization therapy in 87 cases of acute ischemic stroke due to a major intracranial arterial occlusion. Of them, we identified three cases having concomitant $\mathrm{SDH}$ at presentation.

\section{Clinical Presentation}

Case 1. A 67-year-old right-handed man was admitted to our hospital complaining of left extremity weakness after 70 minutes from stroke onset. The initial neurological examination revealed drowsiness, global aphasia, left homonymous hemianopia, and left hemiparesis including facial palsy with an NIH Stroke Scale (NIHSS) score of 19. He also showed a laceration to the right forehead with perilesional swelling.

Case 2. A 72-year-old right-handed man was admitted to our hospital within 3 hours after experiencing acute left-side weakness and dysarthria. The neurological examination showed moderate dysarthria, left hemiparesis including facial palsy, and neglect syndrome with an NIHSS score of 10.

Case 3. A 76-year-old right-handed man was transferred to our hospital following CT revealing an acute SDH 6 hours after symptom onset. The neurological examination revealed stupor, quadriparesis, and decreased brainstem reflex with an NIHSS score of 27. Also, he presented with a laceration to the right forehead with perilesional swelling.

\section{Imaging Findings and Treatment}

At baseline CT, we found an acute SDH in cases 1 and 3, which were associated with head trauma during stroke onset, and incidentally found a chronic SDH in case 2 without evidence of early ischemic changes (Fig. 1a-c). Subsequent multimodal MRI was performed. In cases 1 and 2, diffusion-weighted imaging (DWI) showed small fuzzy hyperintensities in the right MCA territory accompanied by decreased perfusion on perfusion-weighted imaging and right MCA occlusion on MR angiography (MRA) (Fig. 1g, h, j, and k). In case 3, DWI showed fuzzy hyperintensites on the bilateral thalamus accompanied by a distal basilar artery (BA) occlusion on MRA (Fig. 1i and j). The above findings necessitated endovascular therapy to rescue salvageable brain tissue.

Under local anesthesia, mechanical thrombectomy (MT), with no adjuvant thrombolytics, was performed according to our previously published protocol [3]. Forced arterial suction thrombectomy (FAST) using the Penumbra reperfusion catheter was successfully done in all cases without any procedure-related complications (Fig. 1p-u). The final recanalization status using the Thrombolysis in Cerebral Infarction (TICI) score was $2 \mathrm{a}$ in case 1,3 in case 2 , and $2 \mathrm{~b}$ in case 3 . The use of antiplatelet or anticoagulant agents was prohibited during the acute stage of stroke because of the

Table 1. Summaries of Clinical and Angiographic Findings in 3 Cases

\begin{tabular}{|c|c|c|c|}
\hline & Case 1 & Case 2 & Case 3 \\
\hline Sex/age & $\mathrm{M} / 67$ & $\mathrm{M} / 72$ & $\mathrm{M} / 76$ \\
\hline \multirow[t]{2}{*}{ Location and characteristics of SDH } & Right supratentorial, & Left frontal, & Right fronto-parietal, \\
\hline & Acute traumatic & Chronic & Acute traumatic \\
\hline Stroke risk factors & Smoking & Afib, HTN, Smoking & Afib, HTN, Smoking \\
\hline Stroke etiology & Cryptogenic & Cardiogenic & Cardiogenic \\
\hline Site of occlusion & Right MCA & Right MCA & BA \\
\hline Endovascular treatment & FAST & FAST & FAST \\
\hline Immediate recanalization & $\mathrm{TICI} 2 \mathrm{a}$ & $\mathrm{TICl} 3$ & $\mathrm{TICI} 2 \mathrm{~b}$ \\
\hline Baseline NIHSS score & 19 & 10 & 27 \\
\hline 7-day NIHSS score & 20 & 0 & 11 \\
\hline 3-month mRS score & 5 & 0 & 4 \\
\hline Onset-to-door time & $70 \min$ & $172 \min$ & $365 \min$ \\
\hline Onset-to-treatment time & $285 \min$ & $330 \mathrm{~min}$ & $481 \mathrm{~min}$ \\
\hline Onset-to-recanalization time & $375 \min$ & $385 \min$ & $521 \mathrm{~min}$ \\
\hline
\end{tabular}

Abbreviations: NIHSS, NIH Stroke Scale; SDH, subdural hematoma; afib, atrial fibrillation; HTN, hypertension; MCA, middle cerebral artery; BA, basilar artery; FAST, forced suction thrombectomy; TICI, Thrombolysis in Cerebral Infarction. 
Mechanical Thrombectomy in Acute Stroke Patients

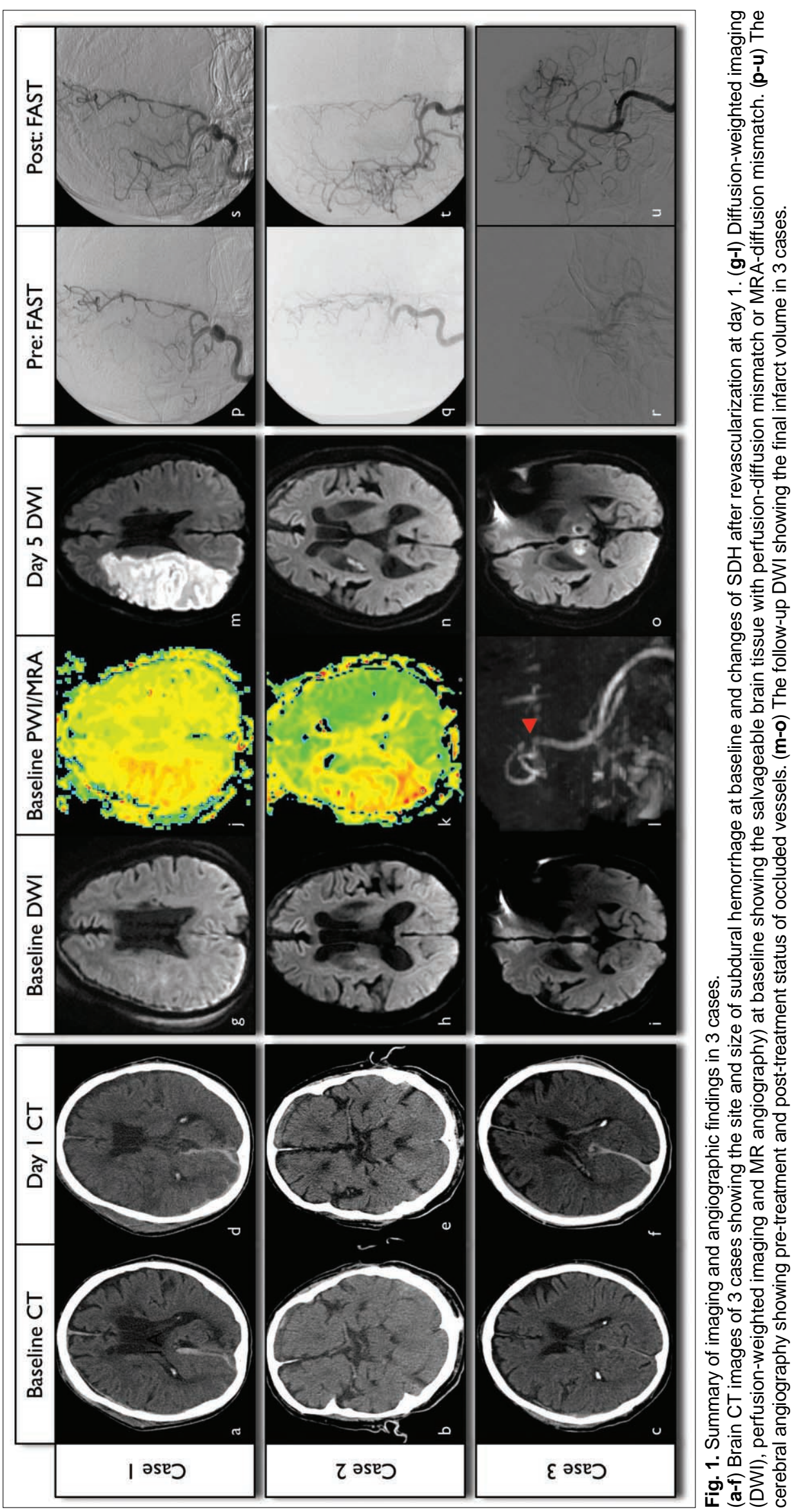


concomitant presence of intracranial bleeding. One day after MT, all three patients were given follow-up brain CT to confirm the safety of MT in the presence of concomitant bleeding (Fig. 1d-f). In cases 1 and 3, there was a small increase in hemorrhage and subdural fluid collection on the contralateral side. In case 2 , there was no change in terms of the SDH. 5-day follow-up DWI was taken to compare with pretreatment DWI (Fig. 1m-o). In case 1, there was a significant increase in infarct volume in the right MCA territory. In cases 2 and 3, the signal change was insignificant.

\section{In-hospital course and clinical outcome}

In case 1 , despite TICI 2 a recanalization, there was a decline in the patient's neurological status during hospitalization, and he was transferred to a rehabilitation center for further recovery. He was completely dependent on others for daily living at three months, and his modified Rankin Scale (mRS) score was 5. In case 2 , he recovered from his neurological deficit completely (NIHSS score of 0 ). The mRS at three months was also 0 . In case 3 , with timely recanalization, his neurological status improved from a baseline NIHSS score of 27 to a 7-day NIHSS score of 11 . However, he suffered from aspiration pneumonia, which prolonged his hospitalization, delaying rehabilitation and recovery. The mRS at three months was 4 .

The summaries of the clinical and angiographic findings are shown in the Table, and the brain CT, MRI, and angiographic findings are shown in the Figure.

\section{DISCUSSION}

Endovascular revascularization therapy is an accepted treatment for selected AIS patients. Timely recanalization of the target vessel in an acute ischemic stroke is associated with a 4- to 5-fold increase in the odds of a good clinical functional outcome and a 4- to 5-fold reduction in the odds of death [4]. Although endovascular therapy is a useful treatment option, the presence of a bleeding risk, such as intracranial hemorrhage or abnormal hemostasis, has been an exclusion criterion in decision-making regarding the use of chemical thrombolytics $[2,5]$. However, the use of MT alone in patients at risk of bleeding may seem feasible and safe. Yet, recently, in an MT trial comparing stent retrievers and Merci devices, imaging exclusion criteria included the presence of intracranial bleeding at baseline $[6,7]$. And a current set of guidelines for endovascular revascularization therapy proposed by a joint panel had exclusion criteria that included intracranial hemorrhage, specifically subdural hematoma [8].

In real clinical practice, we sometimes see patients presenting with AIS and concomitant SDH. According to many published MT trials, these patients are excluded from MT despite a chance of recovery with timely recanalization. In general, SDH is due to bleeding from the cortical bridging veins that drain into the superior sagittal sinus or other dural sinuses. The cortical bridging veins are vulnerable to venous hypertension or tearing in the subdural space following trauma. Theoretically, recanalization using MT devices does not directly affect venous pressure but rather impacts the arterial pressure of affected distal tributaries. So, our case series offers some evidence of safely performing MT in AIS patients with SDH.

The main findings of this case report are as follows: 1) the size of the concomitant SDH was not significantly affected by recanalization with MT; 2) the outcomes seem not to be much affected by the presence of the concomitant SDH, rather by the recanalization status and resultant infarct volume expansion; and 3) MT alone in the three cases was safely implemented in our clinical practice, relative to the presence of SDH. However, more convincing data about MT for patients at risk of bleeding are needed, and a clear guideline indicating circumstances when MT with SDH is safe is desirable.

\section{Acknowledgement}

We thank Wade Martin of Medical Research International for his critical English revision.

\section{References}

1. Jauch EC, Saver JL, Adams HP Jr, Bruno A, Connors JJ, Demaerschalk BM, et al. Guidelines for the early management of patients with acute ischemic stroke: a guideline for healthcare professionals from the American Heart Association/American Stroke Association. Stroke 2013;44:870-947

2. Chalela JA, Katzan I, Liebeskind DS, Rasmussen P, Zaidat O, Suarez JI, et al. Safety of intra-arterial thrombolysis in the postoperative period. Stroke 2001;32:1365-1369

3. Kang DH, Hwang YH, Kim YS, Park J, Kwon O, Jung C. Direct thrombus retrieval using the reperfusion catheter of the Penumbra system: forced-suction thrombectomy in acute ischemic stroke. AJNR Am J Neuroradiol 2011;32:283-287

4. Rha JH, Saver JL. The impact of recanalization on ischemic stroke outcome: a meta-analysis. Stroke 2007;38:967-973

5. Meguro T, Higashi H, Nishimoto K. Acute subdural hematoma after intra-arterial thrombolysis for acute ischemic stroke-case report. Neurol Med Chir (Tokyo) 2005;45:627-630

6. Nogueira RG, Lutsep HL, Gupta R, Jovin TG, Albers GW, 


\section{Mechanical Thrombectomy in Acute Stroke Patients}

Walker GA, et al. Trevo versus Merci retrievers for thrombectomy revascularisation of large vessel occlusions in acute ischaemic stroke (TREVO 2): a randomised trial. Lancet 2013;380:12311240

7. Saver JL, Jahan R, Levy EI, Jovin TG, Baxter B, Nogueira R, et al. SOLITAIRE ${ }^{\mathrm{TM}}$, with the intention for thrombectomy (SWIFT) trial: design of a randomized, controlled, multicenter study comparing the SOLITAIRE ${ }^{\mathrm{TM}}$, Flow Restoration device and the MERCI Retriever in acute ischaemic stroke. Int J Stroke 2012 Nov 6 [Epub ahead of print]

8. Lazzaro MA, Novakovic RL, Alexandrov AV, Darkhabani Z, Edgell RC, English J, et al. Developing practice recommendations for endovascular revascularization for acute ischemic stroke. Neurology 2012;79:S243-55 